\section{DIABETES MELLITUS AND PREGNANCY BY}

C. E. WOODROW, M.B., M.R.C.P.

Honorary Physician, Scarborough Hospital

The following case report illustrates some of the interesting changes that take place in the carbohydrate tolerance of the mother during pregnancy, and stresses the consequent necessity for careful and continuous control during this period to avoid the dangers of acidosis and to correct depletion of the glycogen stores due to any toxaemia which may arise. Since the introduction of insulin the maternal death rate in diabetics has greatly decreased. A survey of the literature shows that in pre-insulin days the immediate mortality rate was as high as $30 \%$, and rose to something approaching $50 \%$ about two years after delivery. The average figure for immediate mortality, with control with insulin, is now $5 \%$, but this is still considered to be at least 6 times greater than for non-diabetic women. These somewhat favourable statistics have been obtained from series in which special care has been exercised, so it is probable that the dice are still very much loaded against the diabetic pregnant woman. The foetal mortality rate still remains very high, and, according to many observers, pre-partum, intra-partum, and post-partum deaths of infants born of diabetic women still continue at approximately $40 \%$. Any method which might reduce this high death rate is worthy of trial, and for this reason early Caesarean section, followed by specialized care of the infant after delivery to prevent hypoglycaemia, as advocated by Prof. Wilder of the Mayo Clinic, was tried in the present case with a successful result. One case is of no use in assessing the value of any particular form of therapy; but I hope the method will be tried by others in a larger number of cases. The necessity for some stabilized method which offers a better chance for the infant is more urgent now that the fertility of diabetic women is far greater than it was.

\section{Case Report}

The patient, a married woman aged 28 , was a known mild diabetic. She came to the out-patient department on Aug. 25, 1941, 5 months pregnant, her urine giving a red reaction to Benedict's test and her fasting blood sugar amounting to $0.206 \%$. She was admitted next day and was not able to leave during the remainder of the pregnancy. The treatment of the case falls under three main headings.

\section{Care of the Mother during Pregnancy}

The essentials are primarily an optimal nutrition with an adequacy of vitamin $\mathrm{B}$ and calcium, and adequate control of the carbohydrate metabolism, due regard being paid to the dangers to both mother and foetus of the development of hypoglycaemia. As pregnancy depresses the renal threshold in patients with established diabetes I found it safer (owing to the difficulties of frequent blood-sugar estimations) to regulate treatment so that the urine almost always contained a trace of sugar. Also, for control it was necessary that 4-hourly urine analyses should be done, for insulin requirements during pregnancy are many and varied. In the present case it is not known what the actual requirements were during the first 5 months (the patient then taking 20 units of protamine-insulin daily), but while under my care these requirements varied between 85 and 25 units a day (given in 2 doses). Broadly one can say that as the pregnancy advanced during the last 3 months, and especially during the lasst 6 weeks, the carbohydrate tolerance increased, so that just before delivery very much less insulin was needed for effective stabilization. This accords with the theory that the pancreas of the foetus in diabetics helps in the maternal demand for insulin in the later months of pregnancy.

The patient only once had severe hypoglycaemia during the initial stages of stabilization. This was at 2 a.m., when she was found by the nurse in coma, sweating profusely and trembling. She was easily brought round by adrenaline, followed by $10 \mathrm{c.cm}$. of $50 \%$ glucose intravenously. The coma had occurred during sleep without any warning to the patient, and for this reason it is clear that unless very careful watch is kept the dosage of insulin given must be such that there is little danger of hypoglycaemia developing. The diet contained $130 \mathrm{~g}$. of carbohydrate, and was the usual balanced diet - at least 1 pint of milk a day being given in order to ensure an adequate intake of calcium.

It was decided to perform Caesarean section on Dec. 9-i.e., the beginning of the 37th week. Until Dec. 6 there had been no evidence of toxaemia, but on that date a slight trace of albumin was found in the urine, and the next day this had increased in amount and the blood pressure had risen from $120 / 60$ to $180 / 100$; there was also some oedema of the feet. Fluids were restricted and a low protein diet was ordered. On the 8th the B.P. was $148 / 96$; albuminuria had diminished; some bile was present in the urine, but there was no clinical jaundice. The foetal heart sounds were well heard, and it was decided to adhere to the original plan of doing a Caesarean section on the next morning.

\section{Management of Labour and Puerperium}

The routine to be followed in these cases is an early Caesarean section at the 36 th to 37 th week of pregnancy. White has written: "Prolonged difficult labour, normal or induced, has disadvantages for the diabetic. Those of immediate importance are the early exhaustion of the glycogen reserve and the danger of sepsis, the danger of hyperinsulinism, exogenous and endogenous, and the danger of acidosis. The baby has not the vitality of the child of the non-diabetic, and consequently the risk is great of injury from long labour to this large overdeveloped but at the same time flabby child. Furthermore the induction of labour does not answer the problem as well as Caesarean section, because induction is not safe until the cervix is soft, and the cervix may not be soft until after the baby has died." Also, at 36 weeks labour may fail to begin even after surgical induction, and, in addition, surgical induction introduces an added risk of sepsis.

Consequently, in our case, at 9 a.m. on Dec. 9 a classical Caesarean section was carried out by Dr. M. I. Ealing under general anaesthesia (gas-oxygen, and the very minimum of ether) given by Dr. Roxburgh. Pre-operative treatment was that given for any other surgical operation on a diabetic-viz., half the usual morning dose of insulin and no breakfast. The operation was uneventful and there have been no surgical complications. The B.P. steadily fell until on Dec. 14 it was $110 / 70$ The insulin requirements were based on routine bloodsugar estimations for the first four days after operation, but there was never any anxiety, and 15 units per dose was the maximum needed. Since Dec. 16 the patient has been adequately controlled on a morning dose of 15 units and 10 units at night on a diet containing $130 \mathrm{~g}$. of carbohydrate. All evidences of toxaemia have gone-blood pressure and blood urea normal and no albuminuria.

\section{Management of Infant}

The principal difficulty in these cases has apparently been the death of the infant soon after b:rth, and I am inclined to agree that most likely this is due to hypoglycaemia. There is evidence that the foetal pancreas aids the maternal demands for insulin during the later months of pregnancy ; if this is so, then at birth the foetal pancreas is geared up to a much higher pitch than is necessary-i.e., far in excess of the requirements of the infant alone. There is slight evidence for this view in the present case ; for an estimation of the sugar in the cord blood at birth gave a figure of $0.128 \%$, whereas, despite continuous feeding with glucose (as described iater), the child's blood sugar at 4 p.m. the day after was onlv $0.08 \%$. The treatment of the child was based on this supposition, and it certainly worked well in the present case.

At birth the child was very blue, and the following restorative measures had to be taken before it responded: $(a)$ aspiration of mucus and amniotic fluid from the pharynx and trachea : (b) inhalation of pure carbon dioxide followed by a mixture of $10 \% \mathrm{CO}_{3}$ and $90 \%$ oxygen; (c) artificial respiration ; and $(d)$ injection of 10 minims of coramine followed by $0.5 \mathrm{c} . \mathrm{cm}$. of lobeline into the cord. When breathing normally it was immediately given $5 \mathrm{c.cm}$. of $10 \%$ dextrose solution into each buttock. This dose was given - either orally or by injection-2-hourly for the first 48 hours ( 5 iniections were necessary). During this time no muscular twitchings were observed and there was practically no cyanosis, so any tendency to hypoglycaemia had been combated by these measures. After the first 3 days the dextrose solution was changed to Nestlé's milk and attempts at breast-feeding were started. These had to be discontinued, as the mother's milk was not adequate either in quantity or in quality.

The child was treated as for prematurity. and intranasal oxygen was given intermittently for the first 24 hours. This was not required subsequently, and progress has been uneventful. 
The baby was under constant observation for the first 72 hours of life: should hypoglycaemia develop during this period only prompt treatment with parenteral glucose and oxygen can avert the death of the child.

\section{Summary}

A case of diabetes mellitus and pregnancy is described. The salient features appear to be: (1) The varying carbohydrate tolerance during the pregnancy and the necessity for avoiding hypoglycaemia. (2) Caesarean section as the means of delivery - to be done at the 36th to 37th week of pregnancy. (3) The care of the infant after birth to combat the development of hypoglycaemia, which is the most likely cause of death.

It is thought that by the means adopted the dangers to both mother and child normally associated with pregnancy in a diabetic can be minimized.

I should like to express my indebtedness to Dr. M. I. Ealing for the interest she has taken in this case and for performing the Caesarean section; to Dr. Barbara H. Fiddian, without whose conscientious co-operation the case would not have turned out to be the success it has; and to the Sister and Staff Nurse.

BIBLIOGRAPHY

Barns, H. H. F. (1941). J. Obstet. Gynec., 48, 707.

Skipper, E. (1933). Quart. J. Med., 26, 353.

White, P. (1935). Surg. Gynec. Obstet.,61, 324.

- (1937). Amer. J. Obstet. Gynec., 33, 380.

Titus, R. S., Joslin, E. P., and Hunt, H. (1939). Amer. J. med. Sci., 198, 482. Wilder. R. M. (1940). Clinical Diabetes Mellitus and Hyperinsulinism, 1st ed., Philadelphia.

\section{Medical Memoránda}

\section{Abdominal Pregnancy : Delivery of a Living Eight-months Baby}

The following case is interesting and rare enough to warrant publication.

\section{CASE REPORT}

The patient, aged 41, was admitted to hospital on Aug. 12 1941 , with a history of irregular slight losses almost every week since her last normal period at the end of April. She had had one living child after a normal confinement 20 years previously. She also had some difficulty in micturition. Examination revealed a distended bladder, confirmed by catheterization. On vaginal examination a soft swelling was found in the pouch of Douglas in which ballottement could be obtained, and the cervix was displaced upwards and forwards behind the pubes. A diagnosis of retroverted gravid uterus was therefore made. After a few days of catheterization and lying over on her abdomen, she was able to pass urine normally, and the uterus could be felt abdominally, rising out of the pelvis. The swelling in the pouch of Douglas, in which foetal parts could be felt, remained and could not be displaced, but the cervix became normal in position. She had no further loss P.V., and she was discharged on Aug. 21 to attend the ante-natal clinic as a case of sacculation of a retroverted gravid uterus. She was then nearly 4 months pregnant.

She was last seen at the ante-natal clinic on Sept. 19, when the uterus appeared to be enlarging normally, the height of the abdominal swelling being at the level of the umbilicus. On vaginal examination the swelling filling the pouch of Douglas was still present, and was still thought to be a sacculation of the posterior uterine wall. The patient did not attend the antenatal clinic again as she decided to have her baby at home, in spite of being advised that she should be delivered in hospital.

She was readmitted to hospital on Dec. 31 with a history of red loss P.V. for 2 days and of having passed two small pieces of tissue that morning. She was complaining of vague backache. She also said that she had been having abdominal pains on and off for the past three months; these had been severe enough to keep her in bed at times. On examination the abdomen was enlarged to nearly full term. The foetal parts were very easily felt, with the limbs in front, and the foetal heart was heard in the L.O.P. position. Just above the pubes and towards the right iliac fossa was a rather elastic but solid tumour, which felt like a fibroid in the lower part of the anterior wall of the uterus. On vaginal examination the head was found to be well down in the pelvis, and occupying what was thought to be the sacculation of the posterior wall of the uterus very much thinned out. The cervix was displaced forwards and upwards behind the symphysis pubis. The os was one finger dilated, with some tissue presenting through the os.

It was decided that a Caesarean section should be performed so as to avoid the risk of rupture of the thinned-out posterior wall of the lower segment; this appeared to be occupied by the head, which was pushed so low down in the pelvis that it could not be displaced upwards. The cervix, being displaced up behind the symphysis pubis, was unlikely to dilate satisfactorily. Owing to the ease with which the limbs could be felt kicking beneath the abdominal wall, abdominal pregnancy was suggested.

A laparotomy was performed shortly after admission. An abdominal pregnancy was found. The foetus was enclosed in its amniotic sac, which was lying among the intestines towards the left side of the abdomen. The sac was opened and a normal living male child of about 36 weeks and weighing $5 \mathrm{lb} .10 \mathrm{oz}$. was delivered. Attention was next directed to the placenta. The most extraordinary thing was the placental attachment. The placenta, which had formed itself into a globular mass, had a thick pedunculated attachment to the back of the broad ligament in the region of the right tube. It was thus only necessary to divide the pedicle between two clamps to deliver the placenta. The lower portion of the amniotic sac was adherent to the sigmoid colon and the rectum, and it would have caused a lot of haemorrhage to separate this. It was therefore decided to leave the lower part of the sac and drain it. The uterus was found to be enlarged to about the size of a 14-weeks pregnancy. This was the tumour that was felt towards the right iliac fossa. The operation was completed with the loss of hardly any blood.

The mother made an uninterrupted recovery. The baby also did very well, being discharged from hospital with his mother 25 days after delivery weighing $6 \mathrm{lb}$. $-6 \mathrm{oz}$. over its birth weight. The baby was still living and well when last seen on April 23, weighing $12 \mathrm{lb}$. $8 \frac{1}{2} \mathrm{oz}$. at 16 weeks, and was being breast-fed, with complementary feeds.

\section{COMMENT}

This was apparently a case of secondary abdominal pregnancy, resulting from rupture of a right tubal pregnancy at an early stage, the intact amnion with the enclosed foetus escaping into the abdominal cavity and continuing its growth. The case is unique in having such a pedunculated attachment of the placenta to the broad ligament, making its separation so easy by simply dividing the pedicle between two clamps and thus resulting in very little loss of blood. The tissue passed by the patient was a decidual cast from the uterus.

I wish to thank Mr. E. S. Clayton, medical superintendent, for permission to publish this case and for his help with it.

Christia F. Lucas, M.B., Ch.B.

Resident Obstetric Officer, Manor Hospital, Walsall.

\section{Hyperplasia of the Gums due to Dilantin}

Cases of hypertrophy of the gums after prolonged administration of sodium diphenyl hydantoinate (known as dilantin or epanutin) for the treatment of epilepsy have been reported in the U.S.A., but not, apparently, in this country, where it is possible that they may have occurred but not have been associated with the drug.

The case now described is that of Miss X, aged 26, sent to this hospital for dental treatment. There was gross hypertrophy of the gums of the maxilla only, half of the tooth crowns being covered with hard fibrous nodular masses, with no tendency to bleed and no discomfort except where the interdental papillae have overgrown between the teeth so that mastication is painful. The colour is that of normal gum. The medical registrar of the West End Hospital for Nervous Diseases was good enough to tell me that the patient has been on dilantin gr. 1 t.d.s. since May, 1939, and first complained of gingivitis in Feb., 1940. Some carious molars have been extracted, and radiographs show some apical infection of other teeth.

Cases reported in America usually have had the gums of both jaws affected. A suggested scorbutic origin is disproved by the fact that ascorbic acid neither prevents the occurrence nor cures the condition in the gums; also the microscopical appearance shows an excess of collagen fibres, as well as hyperplasia of the corium, together with that of the covering epithelium to a lesser degree. Haemorrhages are unusual.

It is suggested that careful oral hygiene when the drug is first used may prevent the overgrowth, and that if overgrowth occurs the drug may still be given provided that all sources of infection and irritation are eliminated. Gingivectomy of the grosser masses may be performed, although some writers are of the opinion that the drug must be discontinued if recurrence after excision is to be avoided.

A boy of 11 years attended here some time ago with a similar growth of gums of both jaws; an attempt will be made to follow the case up, as possibly being due to the same drug.

F. ElliotT SMITH, M.R.C.S., L.D.S. Medical Superintendent, Dental School. University College Hospital. 